\title{
Release and transformation of iron- bound organic carbon in marine sediments under anaerobic microbial reduction
}

\author{
YUNRU CHEN ${ }^{1}$, FENGPING WANG ${ }^{1,2^{*}}$
}

${ }^{1}$ State Key Laboratory of Microbial Metabolisms, Shanghai Jiao Tong University, Shanghai 200240, China

${ }^{2}$ School of Oceanography, Shanghai Jiao Tong University, Shanghai 200240, China

*Corresponding: fengpingw@sjtu.edu.cn

In surface marine sediments, about $20 \%$ of the organic carbon $(\mathrm{OC})$ is directly bound to reactive iron phases, which promotes the preservation of organic molecules [1]. However, iron oxides serve as active electron acceptors for anaerobic microbial respiration in marine sediments. This may consequently break the association of iron oxides and organic matter. Studies using synthesized iron-OC coprecipitates [2] and forest soils [3] have revealed that iron-bound OC was mobilized and released during anaerobic microbial iron reduction.

In marine sediments, the fate of iron-bound $\mathrm{OC}$ remains poorly understood. To study this, we incubated marine sediments anaerobically with iron-reducing bacterium Shewanella piezotolerans WP3. During the 12-day incubation, the iron reduction was observed in experimental groups with WP3 cells. The released OC reached $0.5 \mathrm{mg} / \mathrm{g}$ when incubated for $45 \mathrm{~h}$, which is almost $10 \%$ of the total OC in original sediments. Afterwards, the dissolved organic carbon (DOC) concentration gradually decreased till the end of the incubation, suggesting re-adsorption or co-precipitation of OC. The optical properties of DOC in the incubation supernatants were characterized by Excitation-Emission Matrix fluorescence spectroscopy. The released DOC included both protein-like and humic-like components, but later humic-like DOC was preferentially removed. All of these indicate that the iron-bound OC pool is very active with the presence of iron-reducing bacteria.

Intriguingly, in our samples the fluorescent DOC only contributes little to the total DOC pool. The dynamics of DOC will be further characterized by FT-ICR MS to gain a full picture at the molecular level. The activity of WP3 cells will be quantified by qPCR to constrain the source of release protein-like DOC. The percentage of iron-bound OC in sediments will be determined to track the fate of the removed humic-like OC.

[1]Lalonde et al. (2012) Nature 483, 198-200. [2] Adhikari et al. (2017) Geochim. Cosmochim. Acta 212, 221233. [3] Zhao et al. (2017) Chem. Geol. 464, 118-126 